\title{
MiR-126 impairs the intestinal barrier function via inhibiting S1PR2 mediated activation of PI3K/AKT signaling pathway
}

\author{
Tanzhou Chen *; Haibo Xue; Ruoyang Lin; Zhiming Huang*
}

1 The First Department of Gastroenterology and Hepatology, The First Affiliated Hospital of Wenzhou Medical University, Wenzhou, 325000, China

*Corresponding author: Zhiming Huang, Tanzhou Chen

Mailing address: The First Department of Gastroenterology and Hepatology, The First Affiliated Hospital of Wenzhou Medical University, Address: Nanbaixiang, Ouhai District, Wenzhou, Zhejiang, 325000, People's Republic of China.

Running title: The role of miR-126 in IBD

\section{Acknowledgments}

This work was part of the Program on Wenzhou Science \& Technology Bureau, No: Y20160026.

This study was funded under Zhejiang Provincial Natural Science Foundation, grant number LY17H030010 .

\section{Abstract}

Background Aberrant expression of miRNAs was a critical element in the pathogenesis of inflammatory bowel disease (IBD). This study aimed to explore the involvement and mechanism of miR-126 in IBD.

Methods In this study, the endogenous expressions of miR-126, S1PR2 and S1P in the pathological tissues of patients with IBD were detected using qRT-PCR and western blot assay, respectively. The luciferase reporter gene assay was performed to confirm the targeting regulatory relation between miR-126 and S1PR2. The transendothelial electrical resistance assay was used to measured the value of TEER.

Results The expressions of miR-126, S1PR2 and S1P in the pathological tissues of IBD patients were significantly higher than that of the control group. Moreover, miR-126 overexpression contributed to intestinal mucosal barrier dysfunction in vitro. S1PR2 was a direct target of miR-126, and S1PR2 expression was negatively regulated by miR-126 in Caco-2 cells. However, S1PR2 activated by S1P had the protection effect for the integrity and permeability of intestinal mucosal barrier via a PI3K/Akt dependent mechanism. MiR-126 silencing possessed obvious protective effects on the intestinal barrier function, but these effects could be reversed by JTE-013 or LY294002.

Conclusion MiR-126 down-regulated S1PR2 and then prevented the activation of PI3K/AKT 
signaling pathway, which ultimately could damage intestinal mucosal barrier function.

Keywords miR-126; S1PR2; inflammatory bowel disease (IBD); intestinal mucosal barrier function

\section{Introduction}

Inflammatory bowel disease (IBD) is a kind of chronic intestinal inflammatory disease, including ulcerative colitis (UC) and Crohn's disease (CD)[1], but the etiology of IBD is still uncertain as yet. The prevalence rate of IBD was high in many western countries, with a morbidity of 1-2/1000. China had showing a obvious increased incidence of IBD for nearly a decade, which was the 10-20 times of that of ten years ago. In view of the serious harm of IBD to human health and the high deterioration rate, more and more researchers have interests in its pathogenesis and treatment. The mechanism was hypothesized as that IBD was caused by the interaction of multiple factors, such as environment, heredity, infection and immunity[2].

MicroRNAs (miRNAs) are a class of non-coding RNA molecules, about 20-23 nucleotides long and identified as important transcriptional regulation factors[3]. MiRNAs can regulate the level of target molecules through several different mechanisms, including transcriptional and translational level and gene silencing through directly binding to the 3'UTR of target mRNA[4]. It has been confirmed that the abnormality of miRNAs expression was a critical element in the pathogenesis of human diseases. MiR-126 was found to significantly increase in active UC tissues and contribute to the development of UC via activation of NF- $\kappa B$ signaling pathway[5]. However, the concrete machanism of miR-126 in IBD needs to be discussed and explored further, which is the focus of this study.

Sphingosine-1-phosphate (S1P), a signaling sphingolipid of the cell membrane, could regulate a variety of physiological processes of cells, such as growth, apoptosis, proliferation and migration[6]. The extensive studies verified that S1P was involved in the pathophysiologic process of many diseases, as well as the pathogenesis of IBD[7]. S1P signaling was also served as an important mediator in the process of lymphocyte trafficking[8]. Several publications demonstrated that S1P was closely related to autoimmune disease through S1P receptors (S1PRs)[9]. In previous studies, S1P exerted its biological effects mainly through its receptors, S1P receptors 1-5 (S1PR1-5) belonged to S1PRs. The results of animal model experiments indicated that targeting 
S1P signaling might be a potential therapeutic method for IBD[10]. It is an important regulator of vascular endothelial cell permeability and fluid balance in vivo and is able to enhance endothelial barrier. Recent studies found that S1P could regulate the permeability of vascular endothelial cells and also enhance endothelial barrier function[11]. Moreover, S1PR2 had inhibition on the endothelial barrier function[12], but the role of S1PR2 in IBD has not been reported. Bioinformatics analysis showed the putative binding site of miR-126 and S1PR2, so we aimed to explore that whether miR-126 was involved in the intestinal mucosal barrier dysfunction via S1PR2.

\section{Materials and methods}

\section{Patients}

20 patients with colorectal neoplasms under the endoscopy were recruited, which were regarded as the model group. Normal intestinal mucosa tissues and another 5 patients with benign enteropathy were chosen as control. Our study was approved by the human ethics committee of The First Affiliated Hospital of Wenzhou Medical University.

\section{Cell line and culture}

Caco-2 cells, which were the normal intestinal epithelial cell line, were purchased from iCellBiotechnology Co., Ltd (China). The cells were maintained in RPBM 1640 medium supplemented with $10 \%$ fetal calf serum under controlled temperature of $37^{\circ} \mathrm{C}$ and a humidified $5 \% \mathrm{CO}_{2}$.

\section{Luciferase reporter assay}

According on Genebank online, the fragment of S1PR2 was obtained. The luciferase reporter plasmid was recombined with the 3'UTR of S1PR2. S1PR2-3'UTR vector and miR-126 mimic or miRNA were co-transfected into the cells used Lipofectamine 2000 transfection reagent (Invitrogen, USA) in accordance with the manufacture's instruction. The measurement of the luciferase activity was used with Dual-Luciferase Reporter Assay System.

\section{Cell transfection}

The miR-126 mimic, inhibitor and their negative control (NC) were synthesized by GENEWIZ Biotechnology Co., Ltd (Suzhou, China). Cells were planted in the 96-well plates and maintained for $24 \mathrm{~h}$. Cell transfection was used by Lipofectamine 2000 reagent (Invitrogen) according to the manufacture's instructions. The efficiency of transfection was examined by real-time PCR. 


\section{Real-time PCR}

Oligotex Direct mRNA Micro Kit (QIAGEN) was used to isolate total RNA from cells, then $1 \mu \mathrm{L}$ of RNA was quantitative. Equal amount of RNA was reverse transcribed for the synthesis of cDNA. Real-time PCR was performed with PCR Mix (SYBR Green I) on the Real-time PCR system (ABI 7900 HT). In this study, the primers were synthesized by GENEWIZ Biotechnology Co., Ltd (Suzhou, China). GAPDH was considered as internal control.

\section{Western blot}

With the lysis in RIPA buffer (Solarbio) and centrifugation for $20 \mathrm{~min}$, proteins were extracted from cells. Bradford method was used to detect the quality of the proteins. SDS-PAGE was used to separate the proteins with equal amount, the proteins were then transferred onto PVDF membrane, with the primary antibodies, which were purchased from Sigma (USA), against S1P, S1PR2, ZO-1 and p-AKT at $4{ }^{\circ} \mathrm{C}$ for $24 \mathrm{~h}$, then the membrane was incubated with the secondary antibodies at room temperature for another $1 \mathrm{~h} . \beta$-actin acted as internal control.

Intestinal epithelial barrier model construction and Trans-epithelial electrical resistance (TER) determination

Caco-2 cells were seeded in a Transwell system for monolayers, TER values were measured by an epithelial voltohmmeter ERS-2 (Merck Millipore). TER was measured until the similar values were occurred on three consecutive measurements. The values were calculated with $\Omega \mathrm{cm}^{2}$. When the TER values of the cells reached at least $500 \Omega \mathrm{cm}^{2}$ at $20 \mathrm{~d}$, showing the model was successfully established.

\section{Paracellular permeability measurement}

After intestinal epithelial barrier model was successfully constructed, LPS was added in the Apical side of the model and cultured with the supplementary of $1 \mathrm{mg} / \mathrm{mL}$ FD-4 solution for $2 \mathrm{~h}$. The paracellular permeability was measured by FD -4 flux. A Synergy H2 microplate reader (Bio Tek) was used for the determination of FD-4 signaling.

\section{Statistical analysis}

Data were presented as means \pm SD by statistical analysis of ANOVA software combined with $\mathrm{t}$-test . The value of $\mathrm{P}$ less than 0.05 was considered as statistically significant difference.

\section{Results}

MiR-126, S1PR2 and S1P with high expression in IBD 
The expression of miR-126 in the pathological tissues of IBD patients $(n=20)$ was significantly higher than that of the control group (about 2.8-fold) $(\mathrm{n}=5)$ (Figure 1 A). Moreover, the mRNA expression of S1PR2 was also up-regulated by 1.61-fold in the pathological tissues of IBD patients $(n=20)$ (Figure $1 \mathrm{~B})$, and it negatively correlated with miR-126 expression (Figure $1 \mathrm{C}$ ). And the protein levels of S1PR2 and S1P expression were significantly increased in the pathological tissues of IBD patients $(n=20)$ (Figure 1 D\&E).

\section{S1PR2 was a direct target of miR-126}

The results of bioinfomatics analysis revealed that miR-126 directly bond at a site located within S1PR2 (Figure 2 A). The relative luciferase activity of S1PR2 was significantly suppressed by miR-126 mimic, but the relative luciferase activity was unaffected in miRNC treatment (Figure 2 A). The protein expression of S1PR2 was decreased with miR-126 mimic; conversely, S1PR2 protein was significantly elevated by miR-126 inhibitor (Figure 2 B).

\section{MiR-126 overexpression contributed to intestinal mucosal barrier dysfunction}

LPS was used for the induction of intestinal mucosal barrier dysfunction and caused continuous reduction of TEER in Caco-2 cells at $0 \mathrm{~h}, 1 \mathrm{~h}, 3 \mathrm{~h}, 6 \mathrm{~h}, 12 \mathrm{~h}$ of LPS treatment (Figure $3 \mathrm{~A})$. Moreover, S1P significantly inhibited the decline of TEER induced by LPS, but miR-126 mimic could largely release the effect of S1P on TEER (Figure 3 A). Furthermore, miR-126 mimic also significantly reversed the S1P-mediated decrease of intestinal paracellular permeability of Caco-2 cells (Figure 3 B). S1P enhanced the expression of S1PR2 and ZO-1 protein in Caco-2 cells treated by LPS, but this increase of S1PR2 and ZO-1 was completely reversed by miR-126 mimic (Figure $3 \mathrm{C}$ ).

\section{S1P regulated intestinal barrier function via modulation of SP1R2}

Si-SP1R2 or JTE-013, an antagonist of S1PR2, could largely release the effect of S1P on the TEER and intestinal paracellular permeability of Caco-2 cells; while CYM-5478, a prototypical agonist of SP1R2, magnified the effect of S1P as shown by increased TEER and decreased intestinal paracellular permeability in vitro (Figure 4 A\&B). In addition, similar results were also observed in the protein expression of ZO-1, p-AKT and AKT. Si-SP1R2 or JTE-013 prominently attenuated the up-regulation of ZO-1 and p-AKT/AKT induced by S1P, but CYM-5478 further promoted the increase of ZO-1 and p-AKT/AKT (Figure 4 C\&D\&E). 
LY294002 treatment decreased p-AKT/AKT as well as the protein expression of ZO-1 (Figure 5 A). CYM-5478 had the obvious effect of promoting p-AKT and ZO-1 at the protein level, but LY294002 almost completely inhibited the increase of p-AKT and ZO-1 protein induced by CYM-5478 (Figure 5 B).

\section{The role of miR-126 in the regulation mechanism of S1P/S1PR2/PI3K/AKT signaling pathway for intestinal barrier function}

TEER of Caco-2 cells was elevated by miR-126 inhibitor, but JTE-013 or LY294002 could down-regulate this increase (Figure 6 A). JTE-013 or LY294002 also reversed the miR-126 inhibitor-mediated repression of intestinal paracellular permeability of Caco-2 cells (Figure 6 B), and eliminated the effect of miR-126 inhibitor on the protein expression of S1PR2, p-AKT, AKT and ZO-1 (Figure $6 \mathrm{C})$.

\section{Discussion}

MiR-126 was convinced to be participated in the genesis and development of ulcerative colitis (UC), but its functional role and mechanism in inflammatory bowel disease (IBD) have not been known well. In the current study, we found that the high expression of miR-126 in IBD had potential destructive effects on the intestinal barrier function via targeted modulation of S1PR2. Furthermore, we also demonstrated that the regulatory mechanism of miR-126/S1PR2 axis was associated with PI3K/AKT signaling pathway.

Our results identified that miR-126 was up-regulated in the pathological tissues of IBD patients, which was consistent with the previous findings. It was also observed that miR-126 significantly increased in active UC group and contributed to the pathogenesis of UC[5]. Altogether, these results indicated that miR-126 may serve as a promotion factor for the progress of IBD. Furthermore, we also found that S1P and its receptor, S1PR2 were increased in IBD patients. S1P, a membrane sphingolipid, can enhance the intestinal epithelial barrier function via activating S1PRs. Additionally, S1PR2 was activated by S1P and promoted the proliferation of cholangiocarcinoma cells[13]. It has been reported that S1PR2 can regulate the migration, proliferation and differentiation of mesenchymal stem cells[14]. Our results suggested that S1PR2 also played an important role in IBD. Further analysis showed that there was a negative correlation between miR-126 and S1PR2 expression in the pathological tissues of IBD, and bioinformatics analysis showed the putative binding site of miR-126 and S1PR2. Considering the 
characteristics miRNAs function, we hypothesized that the role of miR-126 in IBD was mediated by the interactive endogenous relation between miR-126 and S1PR2.

Firstly, the results of luciferase reporter gene assay supported that S1PR2 was a direct target of miR-126 in Caco-2 cells. The overexpression or knockdown of miR-126 directly affected the expression of S1PR2 protein in vitro. Intestinal mucosal barrier dysfunction caused by changes in cell permeability and intracellular junction is a key factor in the pathogenesis of IBD. Tight junction between intestinal epithelial cells is the complex consisting of a series of proteins and fats, which exists widely between cells and cells. The tight junction proteins, the main component of tight junctions, are important for tight junctions to maintain the appropriate structure and function, including Occludin, E-Cadherin, ZO-1. In enterocyte barrier injury model of Caco-2 cells induced by LPS, S1P contributed to stabilize the intestinal barrier function via modulation of SP1R2. However, in the present study, it was strongly confirmed that miR-126 overexpression contributed to intestinal mucosal barrier dysfunction as shown by abolishment of the protection effect of S1P for the integrity and permeability of intestinal mucosal barrier.

S1P-activated SP1R2 could promote cell migration via modulating PI3K/AKT signaling pathway. A great deal of researches showed that PI3K/AKT signaling pathway was an important regulator for intestinal mucosal barrier function in intestinal mucosa injuries. We revealed that S1P/S1PR2 protected intestinal mucosal barrier function via a PI3K/Akt dependent mechanism in vitro. Based on the above results, we further validated the role of miR-126 in the regulation mechanism of S1P/S1PR2/PI3K/AKT signaling pathway for intestinal barrier function. MiR-126 silencing possessed obvious protective effects on the intestinal barrier function.

In conclusion, our results confirmed that miR-126 may serve as a promotion factor for the progress of IBD via targeted modulation of S1PR2. The study provided direct evidence that miR-126 down-regulated S1PR2 and then prevented the activation of PI3K/AKT signaling pathway, which ultimately could damage intestinal mucosal barrier function. Accordingly, miR-126 will be a new therapeutic target for IBD.

\section{References}


1. Rubin DC, Shaker A, Levin MS: Chronic intestinal inflammation: inflammatory bowel disease and colitis-associated colon cancer. Frontiers in Immunology 2012, 3(10):107.

2. Ellinghaus D, Bethune J, Petersen BS, Franke A: The genetics of Crohn's disease and ulcerative colitis - status quo and beyond. Scandinavian Journal of Gastroenterology 2015, 50(1):13-23.

3. Kolacinska A, Morawiec J, Fendler W, Malachowska B, Morawiec Z, Szemraj J, Pawlowska Z, Chowdhury D, Choi YE, Kubiak R: Association of microRNAs and pathologic response to preoperative chemotherapy in triple negative breast cancer: preliminary report. Molecular biology reports 2014, 41(5):2851-2857.

4. Bartel D: MicroRNAs: Target Recognition and Regulatory Functions. Cell 2009, 136(2):215-233.

5. Feng X, Wang H, Ye S, Guan J, Tan W, Cheng S, Wei G, Wu W, Wu F, Zhou Y: Up-Regulation of microRNA-126 May Contribute to Pathogenesis of Ulcerative Colitis via Regulating NF-kappaB Inhibitor IKBa. PloS one 2012, 7(12):e52782.

6. Stunff HL, Galveroperh I, Peterson C, Milstien S, Spiegel S: Sphingosine-1-phosphate phosphohydrolase in regulation of sphingolipid metabolism and apoptosis. Journal of Cell Biology 2002, 158(6):1039-1049.

7. Clemons B, Dedman H, Powell R, Martinborough E, Timony G, Peach RJ, Scott FL: 358 A Small Molecule S1P1 Receptor Agonist With Significant Efficacy in Animal Models of Inflammatory Bowel Disease (IBD). Gastroenterology 2012, 142(5):S-84.

8. Bréart B, Ramosperez WD, Mendoza A, Salous AK, Gobert M, Huang Y, Adams RH, Lafaille JJ, Escalantealcalde D, Morris AJ: Lipid phosphate phosphatase 3 enables efficient thymic egress. Journal of Experimental Medicine 2011, 208(6):1267-1278.

9. Matloubian M, Lo CG, Cinamon G, Lesneski MJ, Xu Y, Brinkmann V, Allende ML, Proia RL, Cyster JG: Lymphocyte egress from thymus and peripheral lymphoid organs is dependent on S1P receptor 1. Nature 2004, 427(6972):355-360.

10. Nixon GF: Sphingolipids in inflammation: pathological implications and potential therapeutic targets. British Journal of Pharmacology 2009, 158(4):982-993.

11. Liu H, Zhang Z, Li P, Yuan X, Zheng J, Liu J, Bai C, Niu W: Regulation of S1P receptors and sphingosine kinases expression in acute pulmonary endothelial cell injury. Peerj 2016, 4:e2712.

12. Blaho VA, Hla T: An update on the biology of sphingosine 1-phosphate receptors. Journal of Lipid Research 2014, 55(8):1596-1608.

13. Liu R, Zhao R, Zhou X, Liang X, Campbell DJW, Zhang X, Zhang L, Shi R, Wang G, Pandak WM: Conjugated bile acids promote cholangiocarcinoma cell invasive growth through activation of sphingosine 1-phosphate receptor 2. Hepatology (Baltimore, Md) 2015, 60(3):908-918.

14. Price ST, Beckham TH, Cheng JC, Lu P, Liu X, Norris JS: Sphingosine 1-Phosphate Receptor 2 Regulates the Migration, Proliferation, and Differentiation of Mesenchymal Stem Cells. International Journal of Stem Cell Research \& Therapy 2015, 2(2).

Figure legends 
Figure 1 The endogenous expression of miR-126, S1PR2 and S1P in the pathological tissues of patients with IBD. The mRNA expressions of (A) miR-126 and (B) S1PR2 in the pathological tissues of IBD patients $(n=20)$ were detected using qRT-PCR, the normal intestinal mucosal tissues $(n=5)$ from patients at the remission phase of IBD as the control. (C) Analysed the correlation between the expression of miR-126 and S1PR2 in the pathological tissues of patients with IBD. (D-E) The protein expressions of S1PR2 and S1P in the pathological tissues and normal intestinal mucosal tissues were measured by western blot assay. $* \mathrm{P}<0.05$, $* * \mathrm{P}<0.01$ vs. control.

Figure 2 MiR-126 could inhibit S1PR2 expression by targeting to S1PR2. (A) The putative binding site of miR-126 and S1PR2 was exhibited and luciferase reporter gene assay was performed to confirm the targeting regulatory relation. (B) The effects of overexpression or knockdown of miR-126 on the expression of S1PR2 protein. $* * \mathrm{P}<0.01$ vs. control.

Figure 3 The effects of miR-126 overexpression on intestinal mucosal barrier dysfunction. Caco-2 cells were treated with $\mathrm{ddH}_{2} \mathrm{O}$ (vehicle group), LPS+S1P, LPS+S1P+miR-126 mimic, LPS, respectively. (A) The TEER and (B) intestinal paracellular permeability of Caco-2 cells were measured. (C) The protein expression of S1PR2 and ZO-1 was determined using western blot assay. $* \mathrm{P}<0.05$ vs. Vehicle, ${ }^{\#} \mathrm{P}<0.05$ vs. LPS, ${ }^{a} \mathrm{P}<0.05$ vs. LPS+S1P.

Figure 4 S1P regulated intestinal barrier function via SP1R2 expression. Caco-2 cells were treated with LPS, LPS+S1P, LPS+S1P+JTE-013, LPS+S1P+si-SP1R2， LPS+S1P+CYM-5478, respectively. JTE-013 is the antagonist of S1PR2, and CYM-5478 is a prototypical agonist of SP1R2. (A) The TEER and (B) intestinal paracellular permeability of Caco-2 cells were measured. (C-E) The protein expression of ZO-1, p-AKT and AKT was determined using western blot assay. $* \mathrm{P}<0.05, * * \mathrm{P}<0.01$.

Figure 5 S1P/S1PR2 regulated tight junction protein ZO-1 through PI3K/AKT signaling pathway. (A) After treatment by LY294002, the protein expression of ZO-1, p-AKT and AKT in Caco-2 cells was measured by western blot assay. (B) After treatment with CYM-5478 or CYM-5478+LY294002, the protein expression of ZO-1, p-AKT and AKT in Caco-2 cells was measured by western blot assay. $* \mathrm{P}<0.05, * * \mathrm{P}<0.01$.

Figure 6 The role of miR-126 in the regulation mechanism of S1P/S1PR2/PI3K/AKT signaling pathway for intestinal barrier function. Caco-2 cells were treated with LPS+S1P, LPS+S1P+miR-126 inhibitor, LPS+S1P+miR-126 inhibitor+JTE-013, LPS+S1P+miR-126 inhibitor+LY294002, respectively. (A) The TEER and (B) intestinal paracellular permeability of Caco-2 cells were measured in different treatment groups. (C) The protein expression of S1PR2, p-AKT, AKT and ZO-1 in the cells was detected using western blot assay. $* \mathrm{P}<0.05$, ** $\mathrm{P}<0.01$. 
A

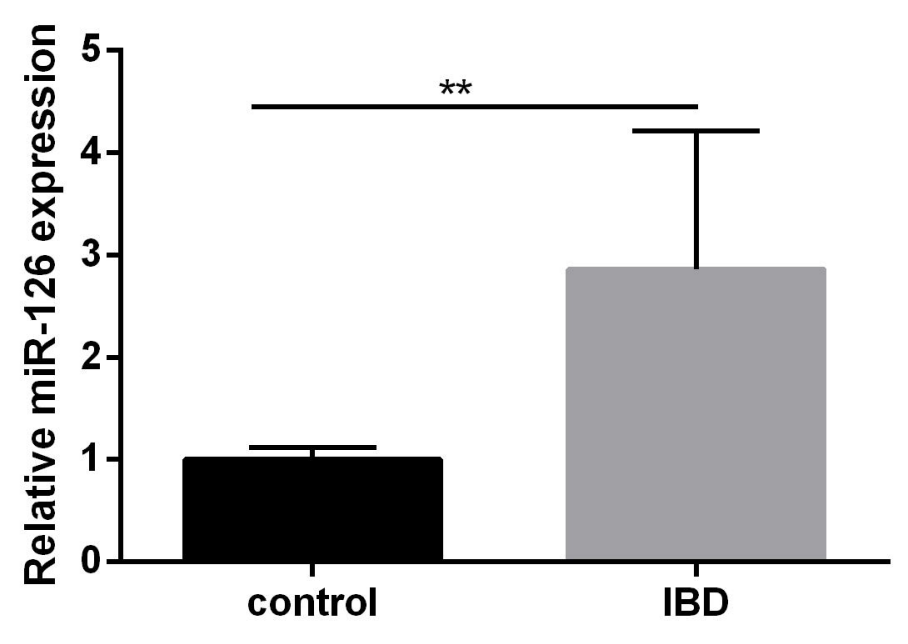

B

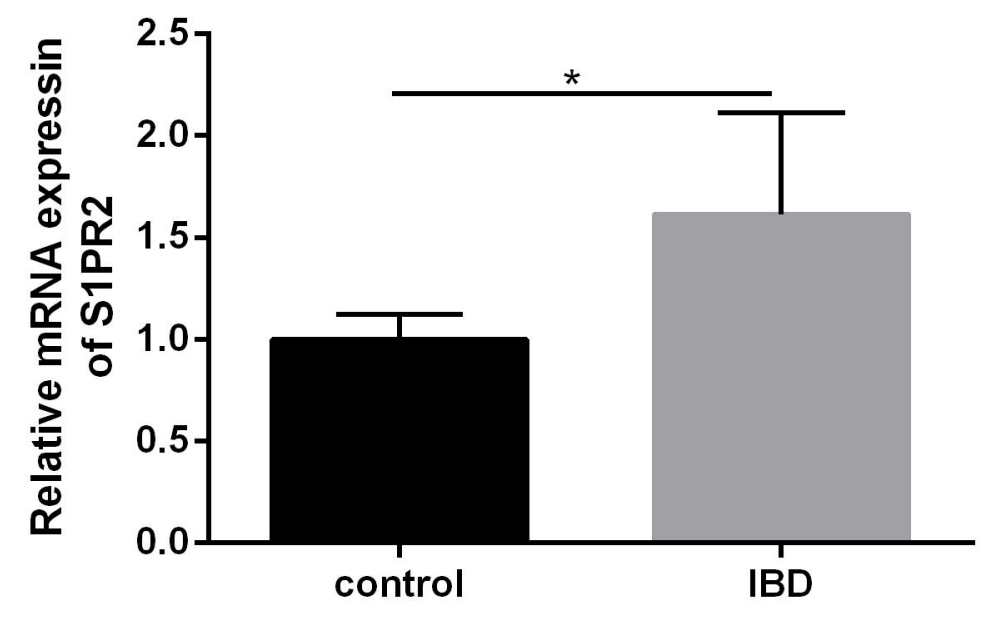

C

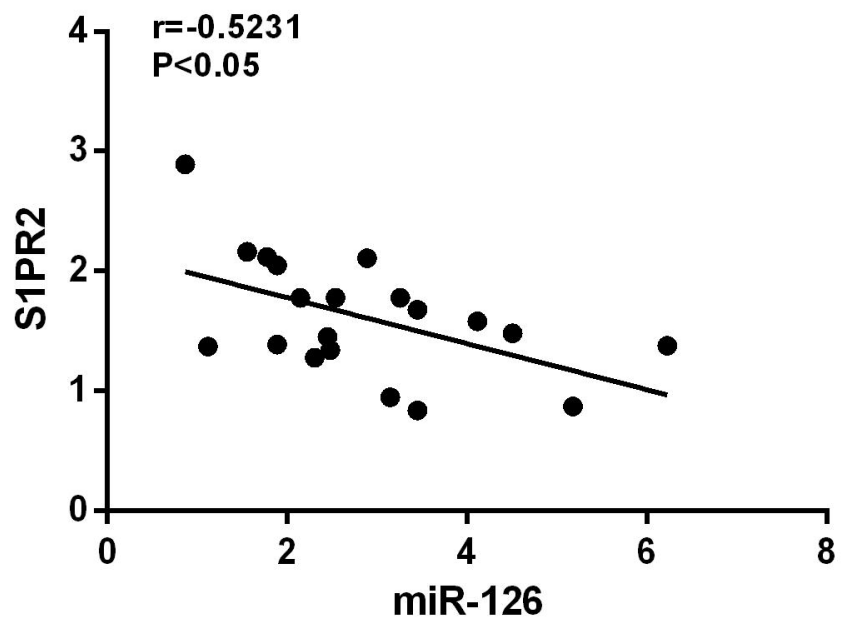

D

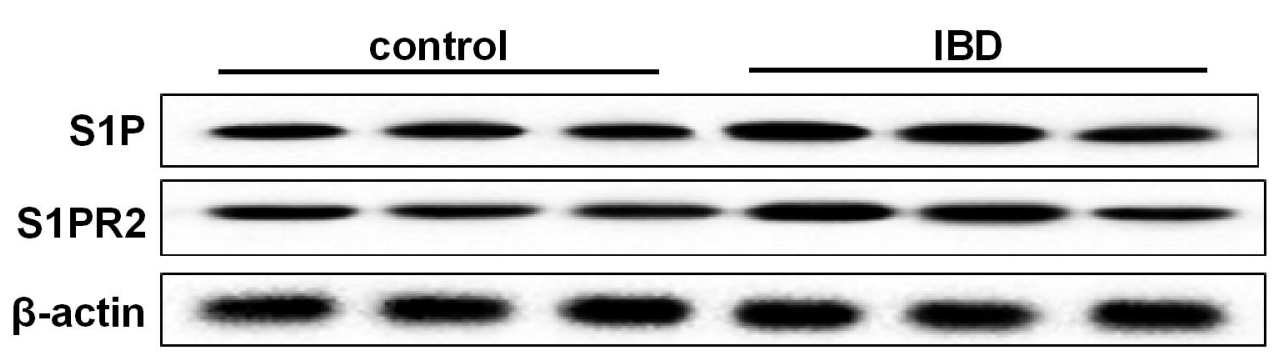

E

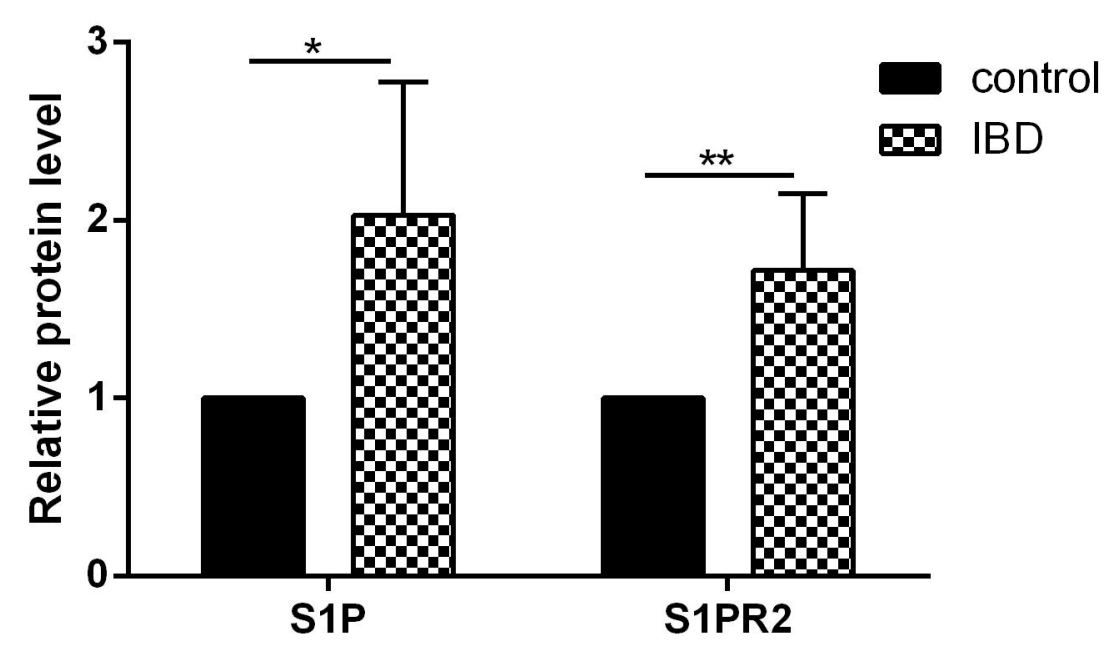


S1PR2 3'UTR 5'...AAUGGAAGGGAUGUUACGGUACA... miR-126-3p 3' GCGUAAUAAUGACUGCCAUGC

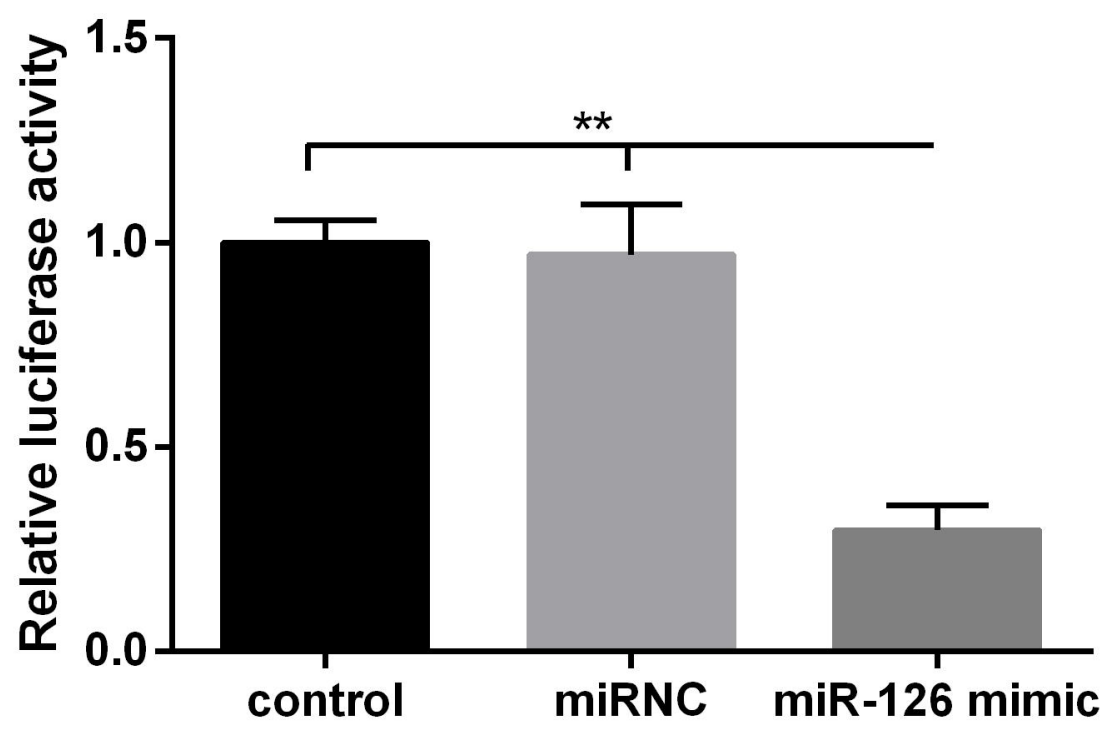

B

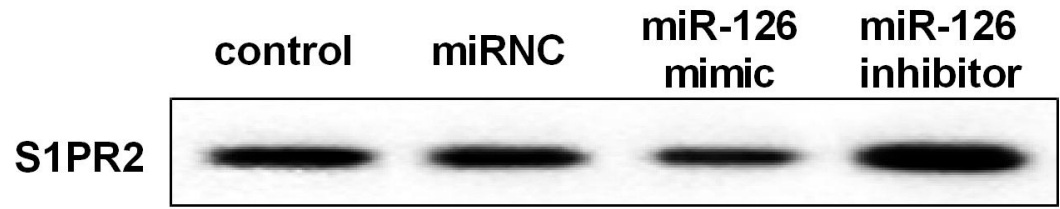

$\beta$-actin

S1PR2 fold

$1 \quad 1.05$

0.78

2.04 


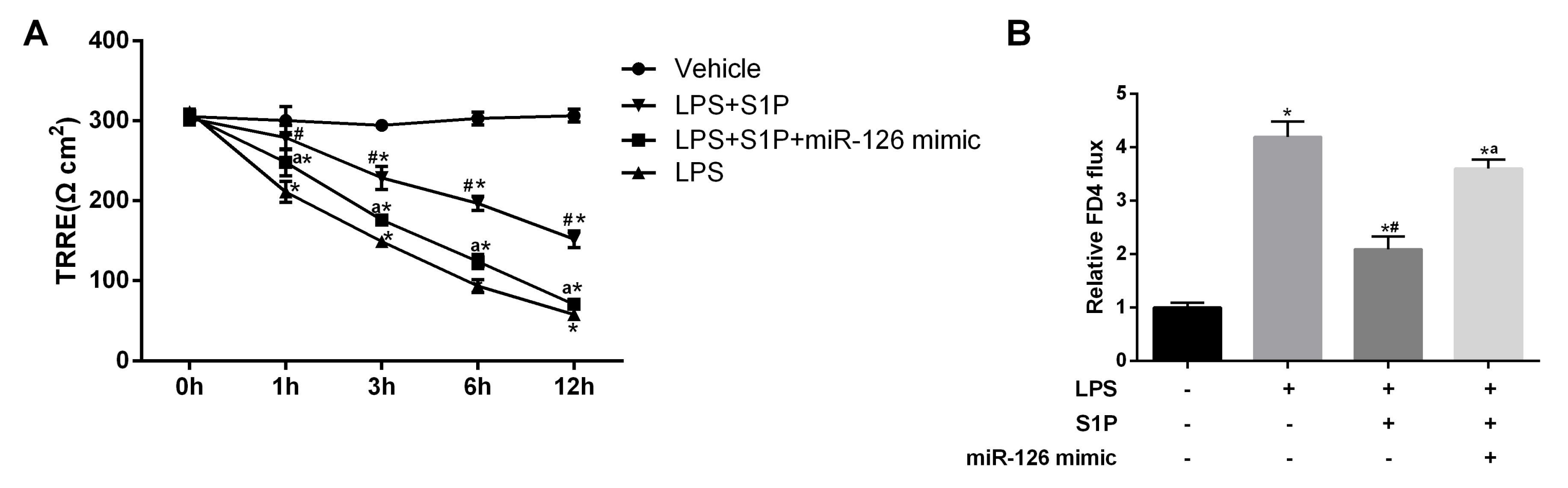

C

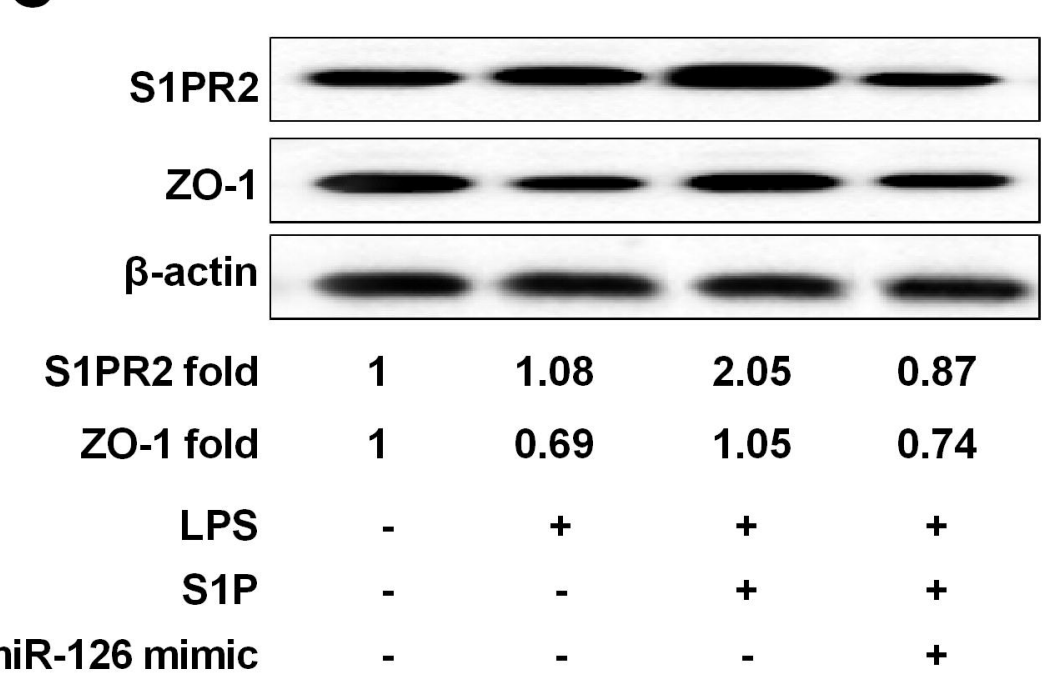


A

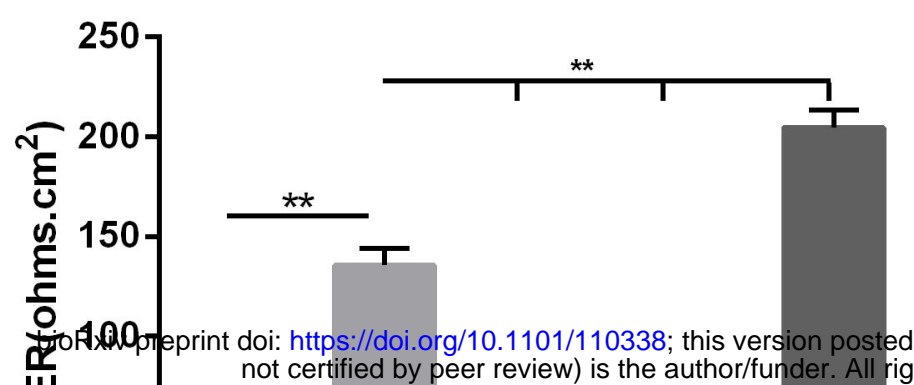

岀

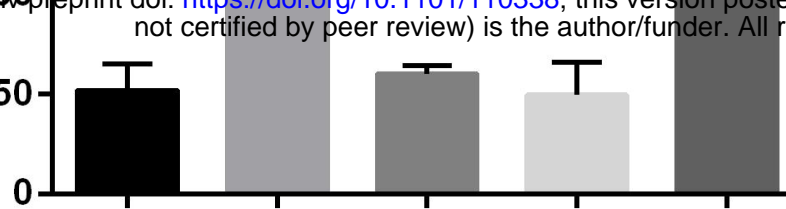

LPS

S1P

Si-S1PR2

JTE-013

CYM-5478

C

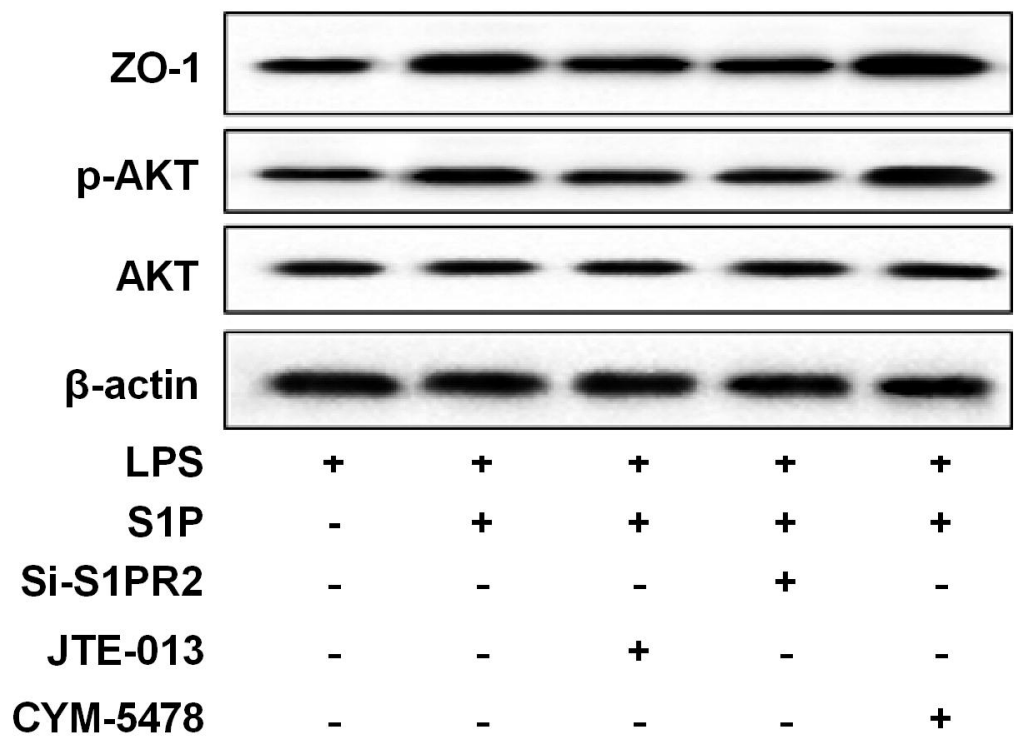

E

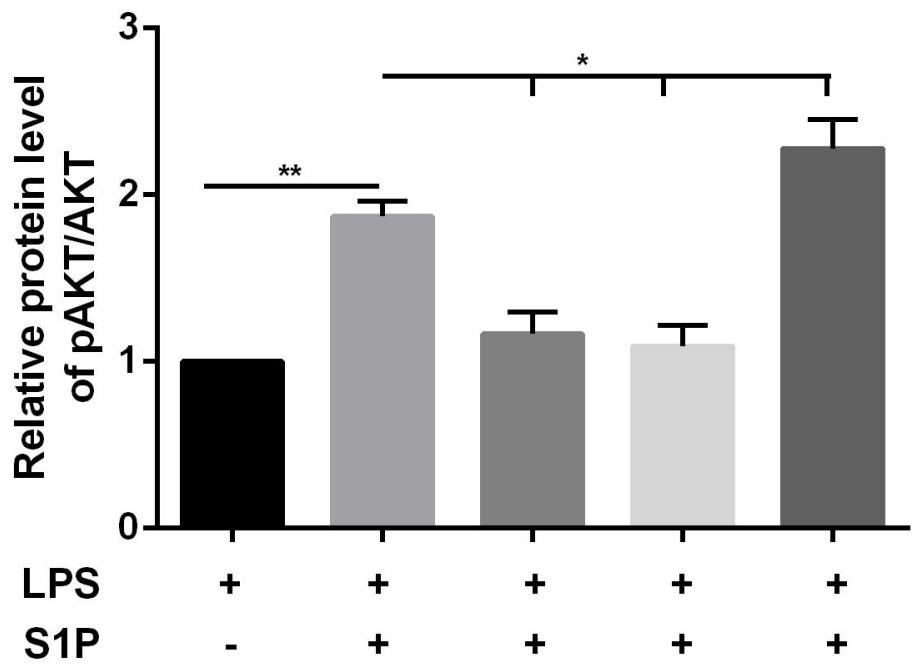

Si-S1PR2

JTE-013

CYM-5478
B

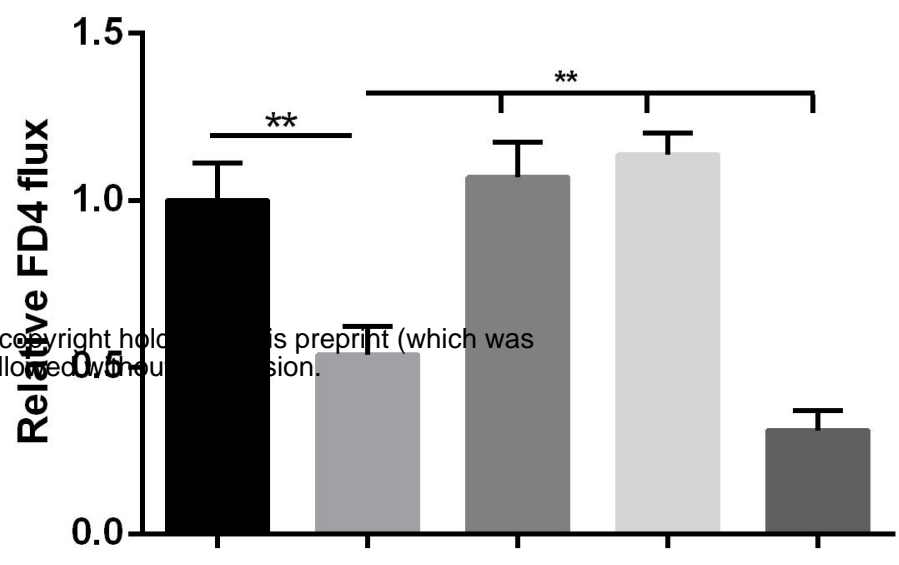

SI-S1PR2

JTE-013

CYM-5478
D

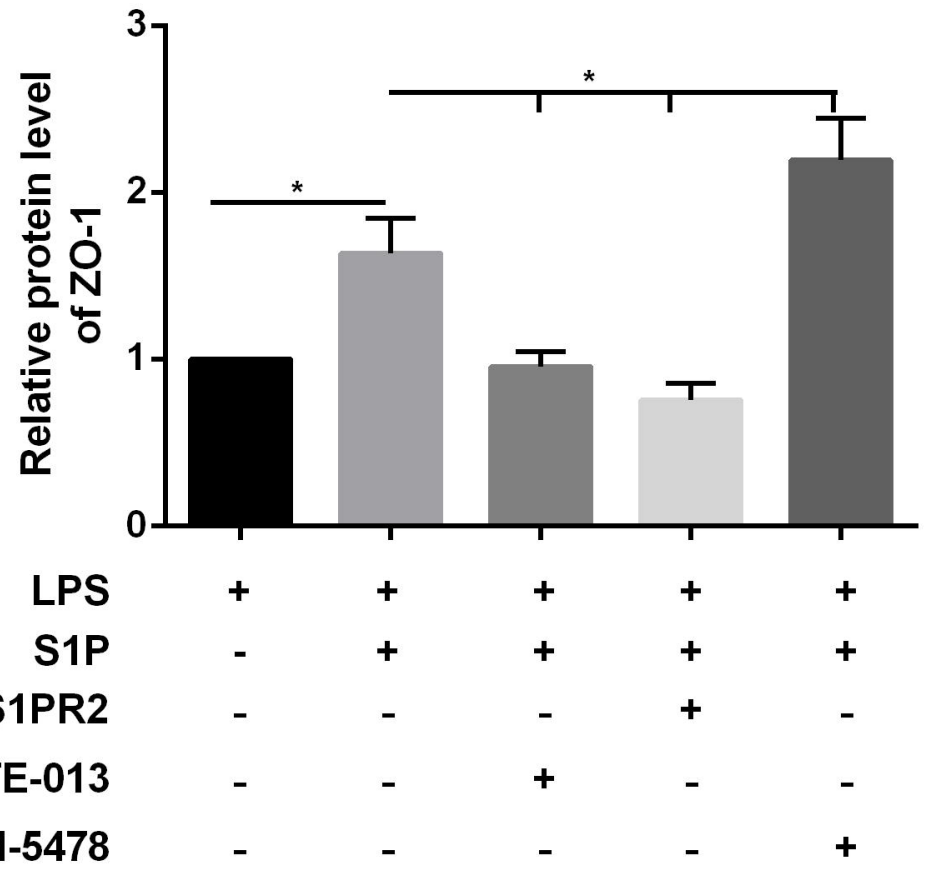

CYM-5478 
A
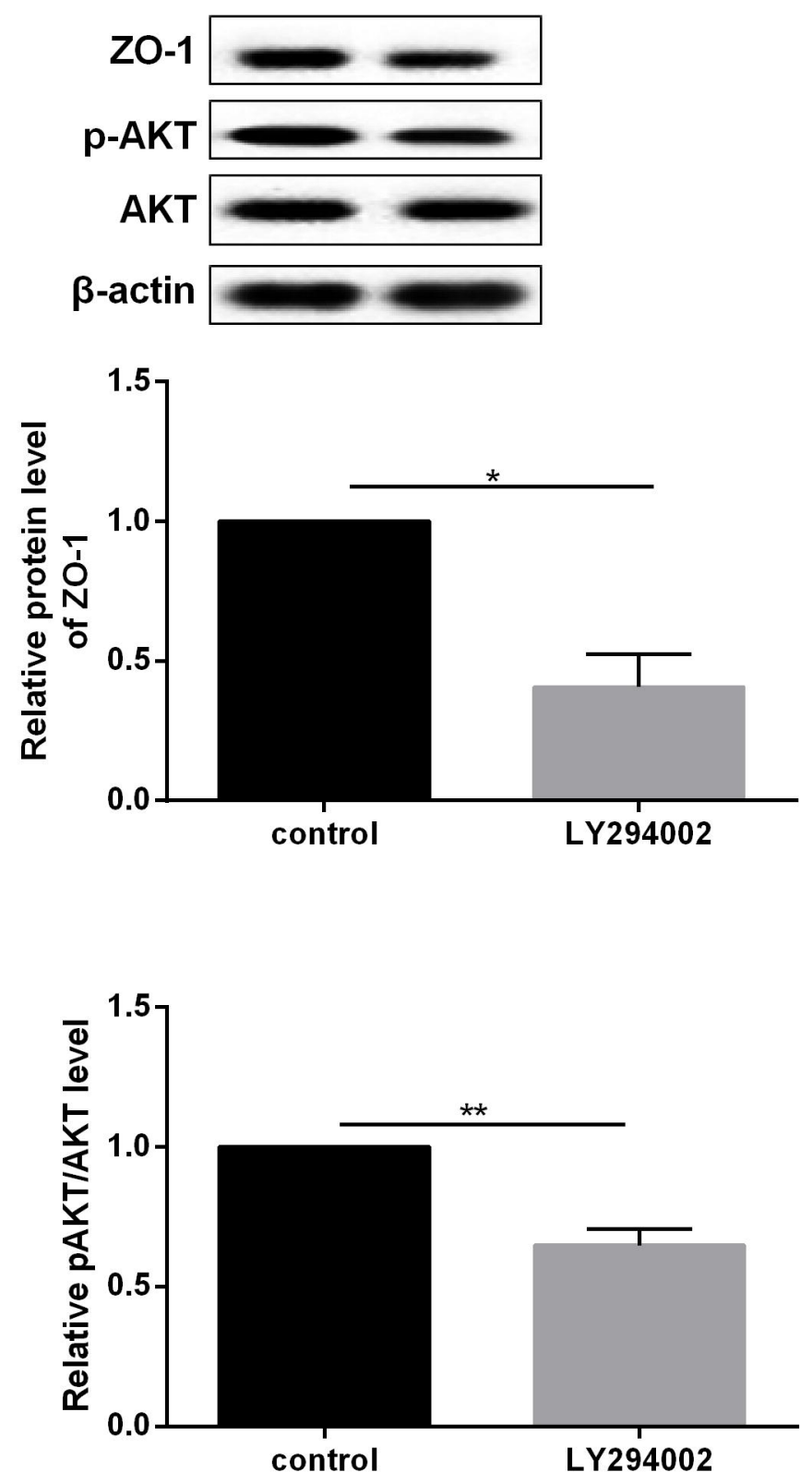

B
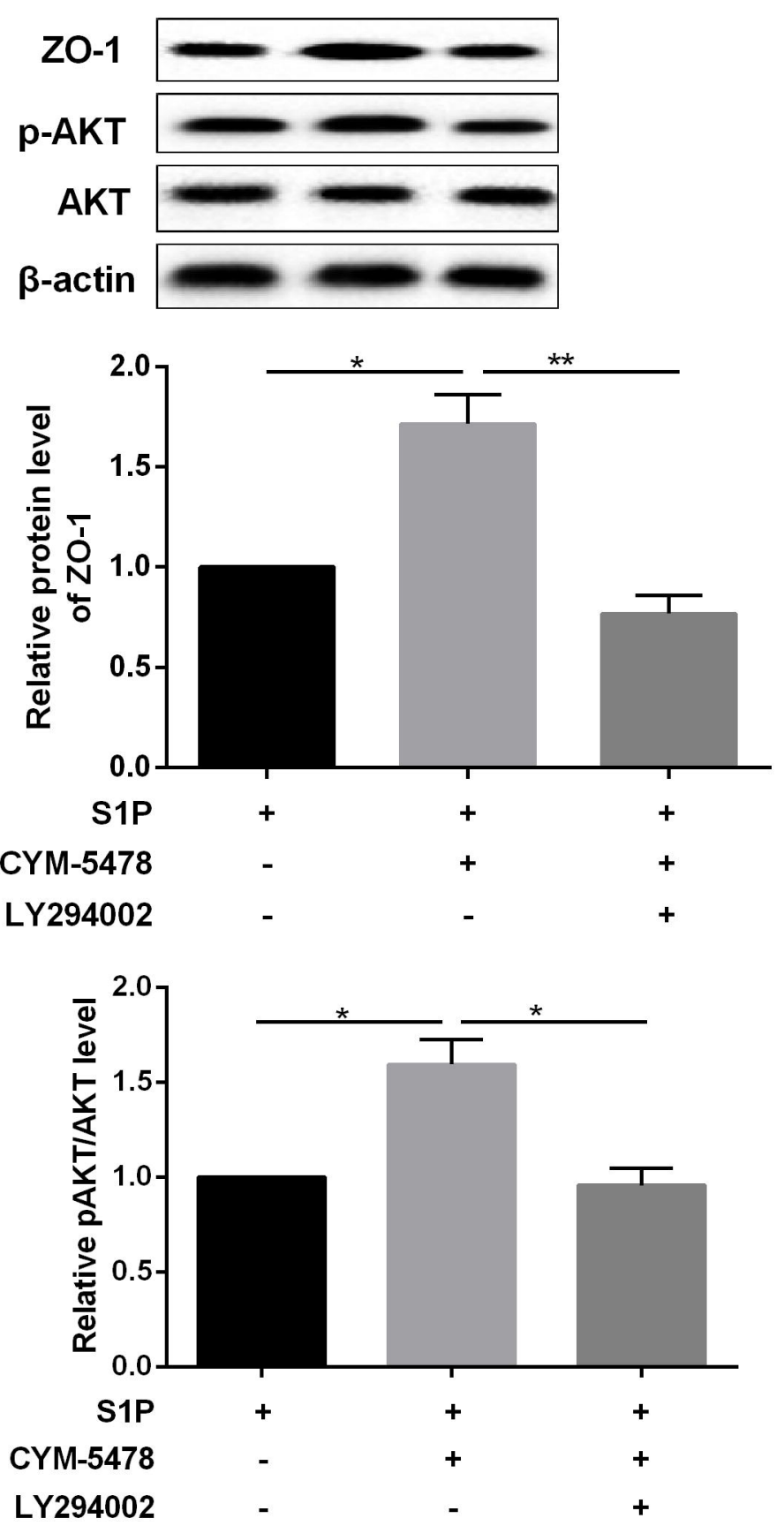

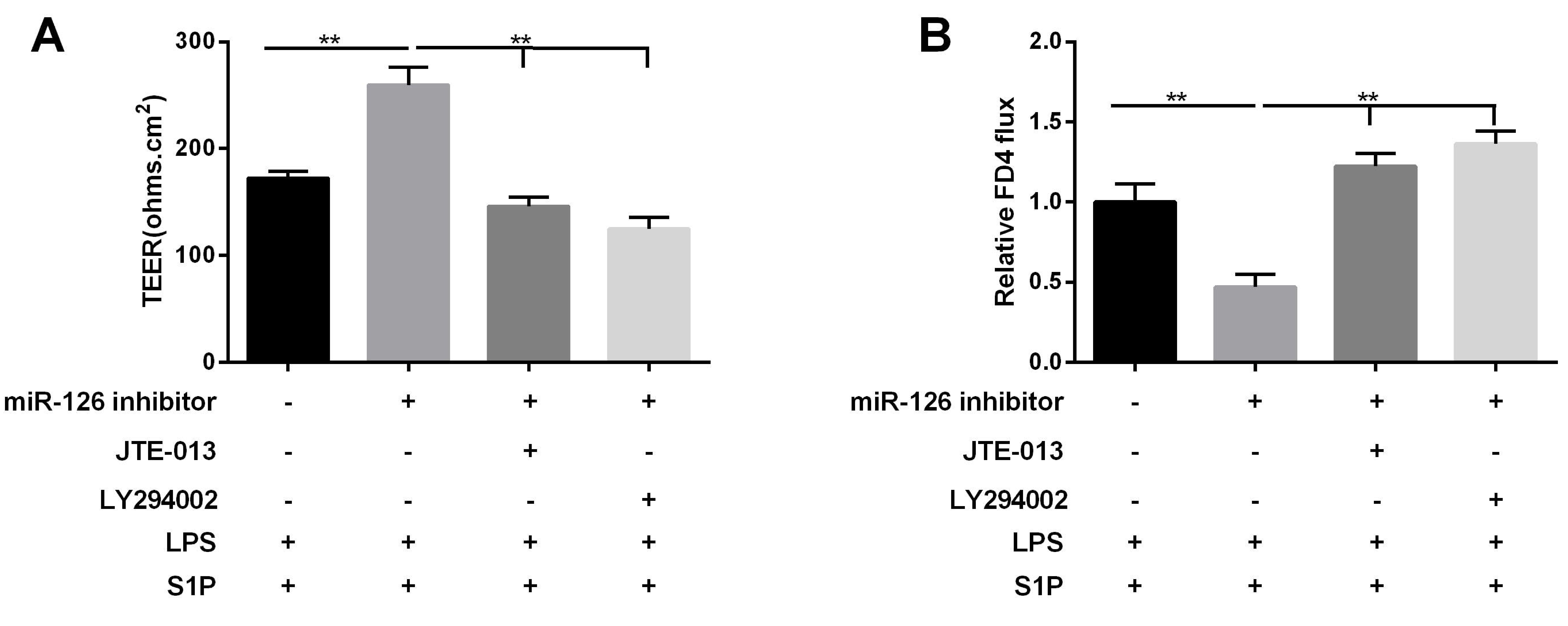

C

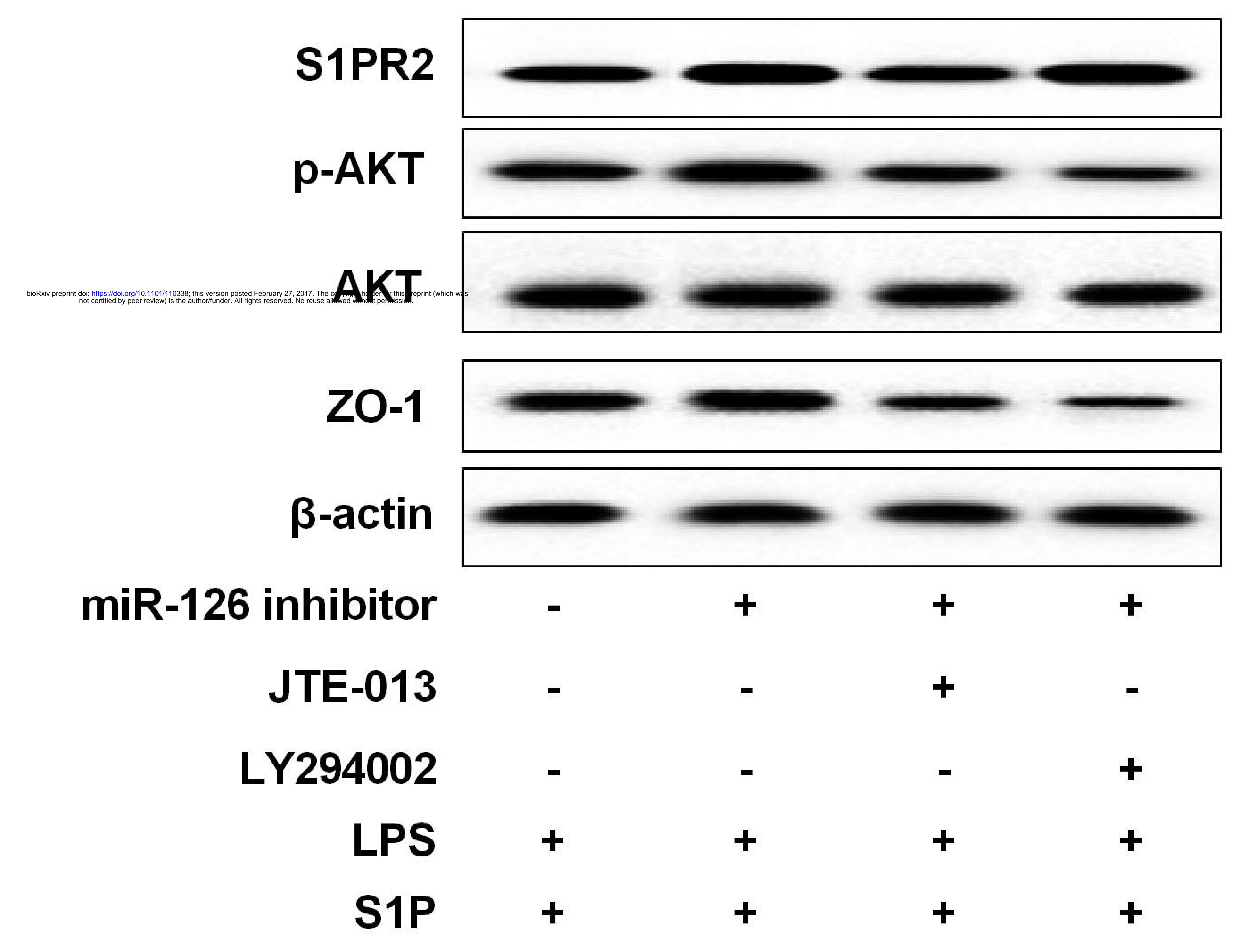

D

- $L P S+S 1 P$

6 LPS+S1P+miR-126 inhibitor

$\square$ LPS+S1P+miR-126 inhibitor+JTE-013

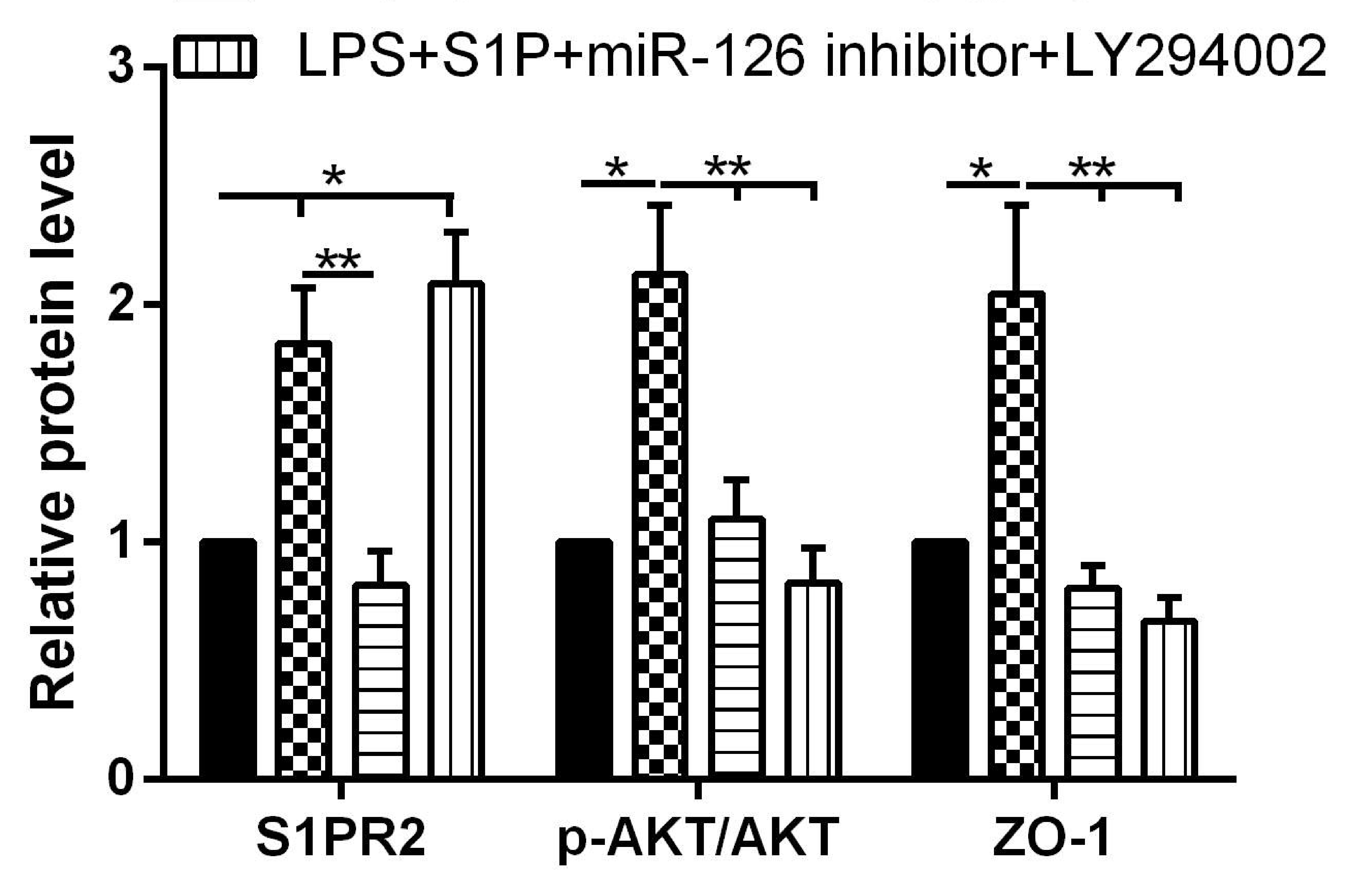

